\title{
Author Correction: REST regulates the cell cycle for cardiac development and regeneration
}

\author{
Donghong Zhang ${ }^{1}$, Yidong Wang ${ }^{1}$, Pengfei Luํ, Ping Wang ${ }^{1}$, Xinchun Yuan ${ }^{2}$, Jianyun Yan ${ }^{3}$, Chenleng Cai ${ }^{3}$, \\ Ching-Pin Chang ${ }^{4}$, Deyou Zheng ${ }^{5}{ }^{5}$, Bingruo $\mathrm{Wu}^{1} \&$ Bin Zhou ${ }^{6}{ }^{6,7}$
}

Correction to: Nature Communications https://doi.org/10.1038/s41467-017-02210-y, published online 07 December 2017.

The original version of this article contained an error in the spelling of the author Jianyun Yan, which was incorrectly given as Jiangyun Yan. This has now been corrected in both the PDF and HTML versions of the article.

Published online: 12 January 2018

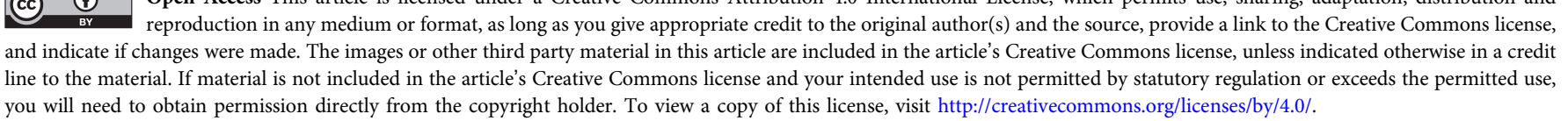

(C) The Author(s) 2017

\footnotetext{
${ }^{1}$ Department of Genetics, Albert Einstein College of Medicine, Bronx, NY 10461, USA. ${ }^{2}$ Department of Medical Ultrasound, The First Affiliated Hospital of Nanchang University, Nanchang 330006, China. ${ }^{3}$ Department of Developmental and Regenerative Biology, The Black Family Stem Cell Institute, and The Mindich Child Health and Development Institute, Icahn School of Medicine at Mount Sinai, New York, NY 10029, USA. ${ }^{4}$ Department of Medicine, Indian University School of Medicine, Indianapolis, IN 46202, USA. ${ }^{5}$ Departments of Genetics, Neurology and Neuroscience, Albert Einstein College of Medicine, Bronx, NY 10461, USA. ${ }^{6}$ Departments of Genetics, Pediatrics, and Medicine (Cardiology), The Wilf Cardiovascular Research Institute, The Institute for Aging Research, Albert Einstein College of Medicine, Bronx, NY 10461, USA. ${ }^{7}$ Department of Cardiology, The First Affiliated Hospital and State Key Laboratory of Reproductive Medicine of Nanjing Medical University, Nanjing, Jiangsu 210029, China Correspondence and requests for materials should be addressed to B.Z. (email: bin.zhou@einstein.yu.edu)
} 